\title{
CRPS diagnosis based on inflammation marker analysis
}

\author{
Cristina Elena Gofita', Anca Emanuela Msetescu', Florentin Ananu Vreju' ${ }^{1}$, Cristin Vere ${ }^{2}$, \\ Mihail Virgil Boldeanu ${ }^{3}$, Otilia Constantina Rogoveanu ${ }^{4}$, Paulina Lucia Ciurea ${ }^{1}$ \\ ${ }^{1}$ Department of Rheumatology, University of Medicine and Pharmacy of Craiova, Romania \\ ${ }^{2}$ Department of Gastroenterology, University of Medicine and Pharmacy of Craiova, Romania \\ ${ }^{3}$ Department of Immunology, University of Medicine and Pharmacy of Craiova, Romania \\ ${ }^{4}$ Department of Medical Rehabilitation, University of Medicine and Pharmacy of Craiova, Romania
}

\begin{abstract}
Objectives. The biological markers that can indicate specifically and sensitively the absence or presence of a certain condition or its state can be used for diagnostic support and disease monitoring. Thus, this research set out to study the changes in neuropeptides (substance $P$ and calcitonin gene-related peptide) pro-inflammatory (TNFa, IL-1 $\beta$ and IL-6) and anti-inflammatory (IL-10) cytokine levels in the blood of patients with CRPS.

Material and methods. Sixty patients were enlisted from the local Rheumatology Clinic of the Emergency County Hospital of Craiova and split into two groups (acute and chronic). CRPS related symptoms were estimated by means of the Neuropathic Pain Questionnaire. The quantification of cytokines and neuropeptides in blood was achieved using the high sensitivity colorimetric ELISA method.

Outcomes. Cytokine analysis led to statistically insignificant results, while for the neuropeptides we obtained significantly increased values in the patient groups. ROC curves were used to assess the diagnostic accuracy of the neuropeptides both returning good AUC values.

Conclusions. Our results indicate that the neuropeptide (substance $P$ and calcitonin gene-related peptide) profile has a good diagnostic sensitivity. A limitation of the study is the low number of patients in the acute group, including more patients in this phase might offer more insights on the cytokines' role, as they could be increased in comparison to chronic patients, due to their short half-life and low acting concentrations.
\end{abstract}

Keywords: complex regional pain syndrome, biological markers, cytokines, neuropeptides

\section{INTRODUCTION}

Complex regional pain syndrome (CRPS) is the modern diagnostic term for the syndrome historically known as causalgia or reflex sympathetic dystrophy (1). It is a chronic neuropathic pain disorder characterized by specific autonomic features and it generally occurs at the level of an extremity after acute trauma. Additional to typical neuropathic pain aspects (allodynia, intense burning pain and hyperalgesia), CRPS is correlated with local edema and changes that suggest autonomic involvement (sweating, altered local skin color and temperature). Other modifications may also occur like trophic modifications to the hair, nails, skin and altered motor function (diminished active range of motion, loss of strength, tremor). The syndrome is separated into CRPS type I which develops without nerve damage and CRPS type II with the presence of documented nerve injury (2). Despite this classic distinction between the two types, signs and symptoms are comparable. Moreover, there is no clear evidence that they are different from a pathophysiologic or treatment responsiveness point of view. It is prevalent in elderly and in the women population $(3,4)$. The most common triggers are crush injuries, fractures, sprains and surgery, though CRPS can basically develop after any (even minor) injury (5). CRPS inflicts not only sever pain but also serious functional deterioration and psychological distress, respectively (6-10). It is possible to overlook CRPS outside specialty 
pain clinics (11). CRPS is one of the most challenging chronic pain conditions to treat effectively, since there is no definitive therapeutic treatment, and most clinical trials have failed to support the efficiency of many generally used interventions (12-15). Considering the absence of alternative viable therapeutic treatments, invasive and costly palliative interventions are frequently utilized, such as intrathecal drug delivery systems and spinal cord stimulation (16).

CRPS diagnosis is based either on the Orlando criteria (2), approved by the International Association for the Study of Pain (IASP), or the reviewed Budapest criteria, which have higher specificity and also include the motor features of the syndrome $(5,17,18)$.

CRPS understanding has increased a great deal recently. Three significant pathophysiological pathways have been described - aberrant inflammatory mechanisms, maladaptive neuroplasticity and vasomotor dysfunction. There is a specific clinical heterogeneity to this condition indicated by the individual variability regarding the activation of these pathways after tissue injury.

Various limited clinical trials indicate that corticotherapy can significantly improve symptoms in some patients suffering from acute CRPS. This suggests the likelihood that inflammatory mechanisms might be involved in CRPS, at least in the acute phase $(19,20)$. Inflammation that contributes to CRPS can develop from two sources. The first one can be represented by usual inflammatory mechanisms. These can contribute via actions of immune cells like lymphocytes and mast cells, which, after tissue damage, produce pro-inflammatory cytokines including interleukin-1 $\beta,-6$ (IL-1 $\beta$, IL-6), and tumor necrosis factor alpha (TNF $\alpha$ ) (21). One of the effects of such cytokines is to enhance extravasation of plasma in the tissue, thereby generating localized edema identical to the one noticed in CRPS. It seems that patients with CRPS also tend to have lower systemic levels of anti-inflammatory cytokines like interleukin-10 (IL-10), which may further support an increased inflammation (22). The second source type could be a neurogenic inflammation, mediated by the release of neuropeptides and pro-inflammatory cytokines straight from the nociceptive fibers in as a reaction to various triggers, that might include even nerve injury (23). The implicated neuropeptide mediators in the neurogenic inflammation involve calcitonin gene-related peptide (CGRP) and substance P (SP) (24). Plasma extravasation and vasodi- lation can both be enhanced by these neuropeptides and thus it can produce the edematous, red, warm limb characteristic in acute CRPS (25). Osteoclasts can also be activated by SP and TNF $\alpha$, thus contributing to the patchy osteoporosis usually observed via $\mathrm{X}$-ray in CRPS patients. Hair growth and increased sweating responses can be stimulated by CGRP both features frequently documented in patients with CRPS $(25,26)$.

The biological markers that can indicate specifically and sensitively the absence or presence of a certain condition or the condition state can be used for diagnostic support and disease monitoring. Thus, this research set out to study the changes in neuropeptides (SP, CGRP) pro-inflammatory (TNF $\alpha$, IL$1 \beta$, IL-6) and anti-inflammatory (IL-10) cytokine levels in the blood of patients with CRPS.

\section{MATERIAL AND METHOD}

\section{Patient and control groups}

Sixty patients were enlisted from the local Rheumatology Clinic of the Emergency County Hospital of Craiova. The procedures and evaluations were also carried out at the Emergency County Hospital of Craiova. The protocol was reviewed and approved by the Ethics Committee of the University of Medicine and Pharmacy of Craiova.

The criteria for inclusion in the study comprised of being older than 18 years of age, with chronic pain from various etiologies (Figure 1), with CRPS features of more than 3 months duration. Diagnosis was applied on standard clinical grounds for pain disorders, including research criteria for CRPS as suggested by the IASP.

Instructions were given to the patients to continue their medications and therapies without any changes for at least a week before study enrollment. This measure was taken due to the concern that interruption would bring severe pain and that the medication withdrawal and rebound symptoms could impede the determination of the patients' typical cytokine activity. Healthy and pain-free control subjects were recruited from the Regional Blood Transfusion Center via consent. Screening for eligibility was accomplished through detailed health questionnaires. Only pain-free and healthy subjects that were not on any treatment were included in the study. The control group included 12 females and 8 males with ages between 20 to 52 years old, corresponding to 
the patients' age range of 23 to 65 years old. The mean height and weight were also comparable (controls: $168 \pm 7 \mathrm{~cm}, 76.5 \pm 10.1 \mathrm{~kg}$; patients: $166 \pm 9$ $\mathrm{cm}, 81.2 \pm 15.3 \mathrm{~kg}$ ), with BMIs in the slightly overweight to overweight range that did not differ substantially (25.2 vs. 29.4).

\section{Assays and procedures}

CRPS related symptoms were estimated by means of the Neuropathic Pain Questionnaire (NPQ) (28). The intensity of pain, determined as average pain over $24 \mathrm{~h}$, and interference related to pain were determined via the Brief Pain Inventory short form (BPI-sf) (29). CRPS sensory signs were evaluated according to the rules of examining the sensory pain specific to the neuropathic pain (2). A decrease or loss of function was noted as negative and allodynia and hyperalgesia as positive phenomena.

Blood was attained from the affected limb by means of venipuncture. Plasma was kept under storage at $-80^{\circ} \mathrm{C}$ until the analysis was performed. Additional to the determinations of cytokines and neuropeptides, routine blood tests were also obtained. These tests included a complete blood count, C-reactive protein (CRP), erythrocyte sedimentation rate (ESR), fibrin (FB), cholesterol (CHL), triglycerides (TGL), total lipids (TL), glycemia (GL) determined by the Emergency County Hospital of Craiova laboratory.

The quantification of cytokines and neuropeptides in blood was achieved using high sensitivity, research purpose, colorimetric ELISA kits (Invitrogen Corp. Camarillo, CA, USA for cytokines and MyBioSource Inc. San Diego, CA, USA for neuropeptides). Samples from thawed specimens (50-100 $\mu \mathrm{L}$ ) were analyzed in duplicate for each subject on a panel that included IL- $1 \beta$, TNF $\alpha$, IL- 6 and IL-10 as well as SP and CGRP. We employed 96-well plates precoated with a monoclonal antibody specific to each neuropeptide or cytokine. An enzyme-linked polyclonal antibody was added only after washing away the unbound substances. After another wash step, the substrate solution was introduced, incubated and then amplified with alkaline phosphatase. The intensity of the color was quantified using an Asys Expert Plus Microplate Reader (Biochrom, Cambridge, UK). The reference curve was employed to compare optical densities and to quantify the samples in $\mathrm{pg} / \mathrm{mL}$. If the values did not fell appropriately within the reference curve, the samples were run di- luted (IL-1 $\beta(3.9-250 \mathrm{pg} / \mathrm{mL}) \mathrm{TNF} \alpha(15.6-1000 \mathrm{pg} /$ $\mathrm{mL})$, IL-6 (2-200 pg/mL), IL-10 (7.8-500 pg/mL), SP (7.8-500 pg/mL) and CGRP (15.625-1000 pg/ $\mathrm{mL})$. All results were above the detection limit.

\section{Data organization and statistical analysis}

The patient data were analyzed as three groups control/reference (R), acute (A) and chronic (C) as follows: 20 patients in each the control and acute groups and 40 in the chronic group. We tested the resulted values for normal distribution using the Shapiro-Wilk test. Since only few of the groups passed the test for normality and we could not correlate them with the ones that did not pass, we could not use the parametric ANOVA test. Instead, to assess the differences between controls, acute and chronic patients, we used the nonparametric Kruskal-Wallis test. In addition, the Mann-Whitney test was used to compare each pair of samples. To determine the discrimination between diseased and normal cases we used the Receiver Operating Characteristic (ROC) curve analysis (the diagnostic performance of a test). The software used to plot the graphs and curves was GraphPad Prism version 8 for Windows, GraphPad Software, San Diego, California USA, www.graphpad.com.

\section{RESULTS}

\section{Patient demographics and characteristics}

Sixty patients were recruited, 20 in an acute CRPS stage and 40 in a chronic state. Clinical etiologies are presented in Figure 1. Symptoms reported by CRPS patients were documented with the NPQ and were considered to be in the range of moderate to higher. These included: aching, burning, electrical, increased pain due to touch and cold temperatures, numbness, stabbing, tingling and sensitive skin. The interference with the highest scores on the BPI-sf included everything related to general activity, enjoyment of life, mood, relationships with others, sleep, walking, work and were ranked within the range of moderate to severe interference. The neurological exam revealed sensory deficits in all patients. Some patients also had positive phenomena of allodynia and hyperalgesia.

Routine blood tests for the patients in the control group were within normal limits (Table 1). The values for the white and red blood cell counts, serum glucose and CRP did not differ significantly between 


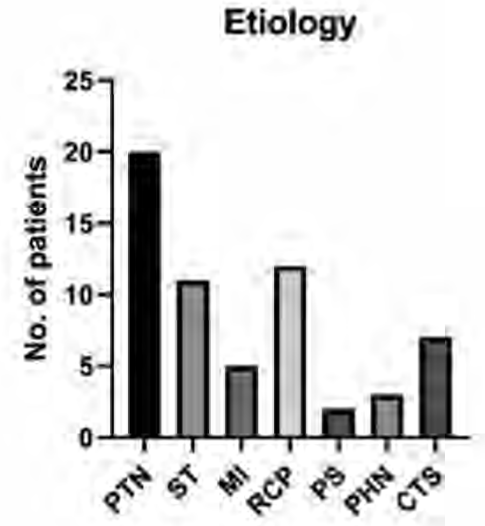

FIGURE 1. Trigger factors for CRPS. PTN: post-traumatic neuralgia, ST: stroke, MI: myocardial infarction, RCP: rotator cuff pathology, PS: paraneoplastic syndrome, PHN: post-herpetic neuralgia, CTS: carpal tunnel surgery

groups. Inflammatory tests (ESR and fibrin) and metabolic markers (cholesterol, triglycerides, total lipids and glycemia) were all moderately increased in patients with pain.
TABLE 1. Mean values of routine blood tests result for the patients with CRPS

\begin{tabular}{|l|c|c|c|c|}
\hline Routine tests & Control & Acute & Chronic & P value \\
\hline ESR (mm/h) & 21.95 & 50.05 & 34.8 & $<0.0001$ \\
\hline CRP (mg/L) & 3.68 & 3.91 & 4.27 & 0.3530 \\
\hline FB (mg/dL) & 351.10 & 435.55 & 420.00 & $<0.0001$ \\
\hline CH (mg/dL) & 195.45 & 217.5 & 256.65 & $<0.0001$ \\
\hline TGL (mg/dL) & 127.5 & 156.05 & 181.97 & $<0.0001$ \\
\hline TL (mg/dL) & 644.25 & 671.05 & 757.87 & 0.0004 \\
\hline GL (mg/dL) & 101.8 & 106.3 & 111.32 & 0.2171 \\
\hline
\end{tabular}

ESR: erythrocyte sedimentation rate, CRP: C-reactive protein, FB: fibrin, $\mathrm{CH}$ : cholesterol, TGL: triglycerides, TL: total lipids, GL: glycemia

\section{Cytokine results}

Circulating levels of the cytokines were not significantly different between the three groups. Even though it seems that the trends of the graphs are particularly consistent with inflammation, like increase levels of TNF $\alpha$, IL-1 $\beta$, IL- 6 and lower concentration of IL-10, the statistical analysis did not show a significant difference between the control and pain patients. Also, a relatively high heterogeneity was observed across all groups.
TNFa

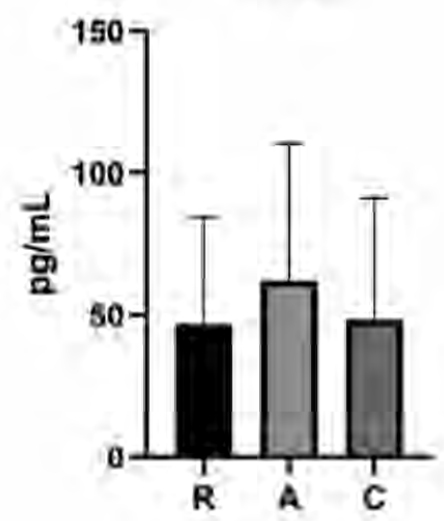

IL-6

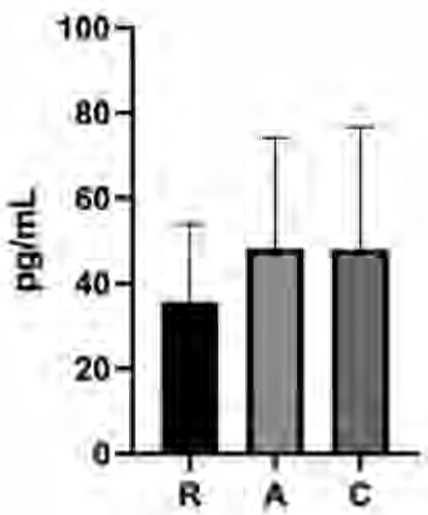

IL-1 $\beta$

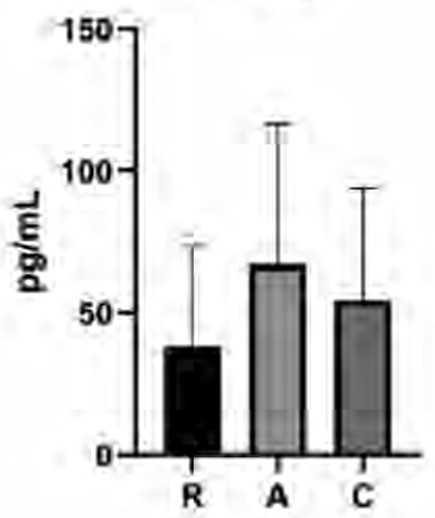

IL-10

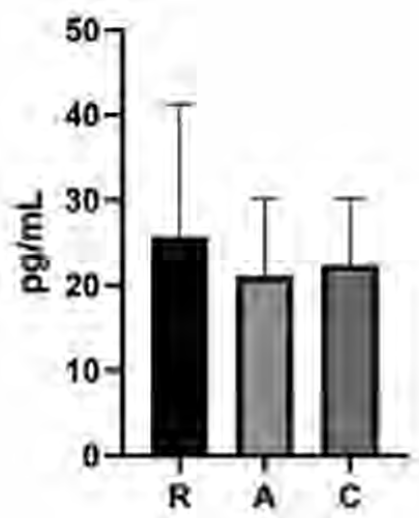




\section{Neuropeptide results}

The neuropeptides returned relevant differences between the control and patient groups $(\mathrm{p}<0.0001)$. As the graphs show the highest mean value was obtained in the acute stage for both SP and CGRP $(p<0.0001)$.
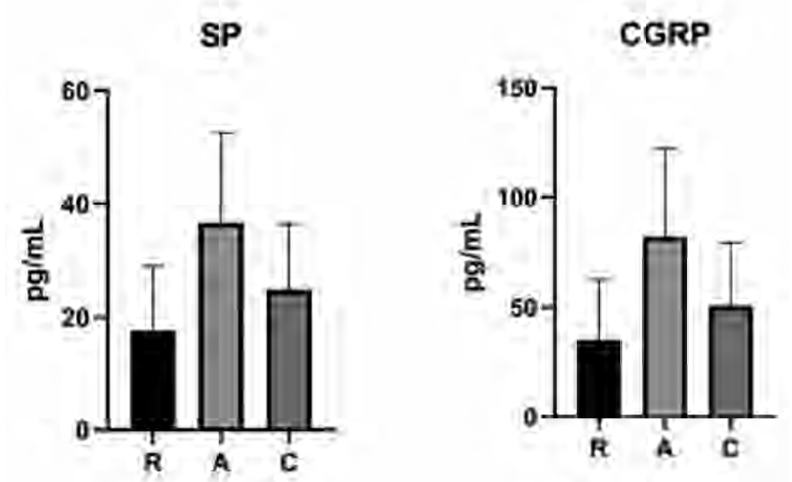

FIGURE 3. Neuropeptides histograms

\section{DISCUSSION}

Even though we observe certain trends (higher levels of proinflammatory (30) and lower anti-inflammatory activity (31)) in the cytokine profiles that we obtained, the results were not statistically significant. The $p$ values for TNF $\alpha$, IL-1 $\beta$, IL- 6 and IL-10 were all increased values $(0.4497,0.0768$, 0.2337 and 0.6300 , respectively) and cannot be used for diagnosis purposes. This was also previously mentioned in other studies (32). An explanation for the poor differences in TNF $\alpha$ groups could be that it acts mostly at low concentrations as a paracrine and autocrine factor (33) and it can be difficult to measure it in the circulation. Probably a more sensitive index for the activity of TNF $\alpha$ could be the assessment of its longer-lasting soluble receptors. IL-6 results also did not reveal any significant differences between control and the affected groups. Being theorized that CRPS is a regional inflammatory process, normal levels of white blood cells, CRP and IL-6 might actually support this, as they represent markers for systemic inflammation rather than local. IL-6 is a pleiotropic cytokine considerably upregulated during various pathologic conditions mostly associated with increased pain and hyperalgesia (34). High serum levels of IL-6 have been identified in patients with autoimmune and chronic inflammatory conditions, burn injuries, malignant tumors, musculoskeletal disorders and various neuropathies. All these disorders have in common a tenderness sensation and hypersensitivity of the affected tissues just like the one observed in CRPS. This information is also supported by our study since the highest values for IL-6, was reported in both of the two patients classified with paraneoplastic syndrome (116 pg/ $\mathrm{mL}$ ) (32).

Slightly higher levels of IL- $1 \beta$ were found in both patient groups. The pro-inflammatory cytokine IL- $1 \beta$ is generated under pathological conditions that are correlated with hyperalgesia and increased pain similar to neuropathies, chronic inflammatory diseases (rheumatoid arthritis) or tumor growth $(35,36)$. Under these conditions, IL- $1 \beta$ can originate from various cell types, like endothelial cells, fibroblasts, mononuclear cells, Schwann cells, and synoviocytes $(34,36)$.

A cytokine with effective anti-inflammatory properties is represented by IL-10. It inhibits the expression of pro-inflammatory cytokines such as IL1, IL-6 and TNF- $\alpha$. Additionally, IL-10 can up-regulate endogenous anti-cytokines and down-regulate pro-inflammatory cytokine receptors. Recent clinical studies indicate that low concentrations of IL-10 could be the key to chronic pain since low levels of this cytokine was found in patients suffering from this debilitating illness (31). Though our results were not statistically significant and cannot be used for diagnosis we can still notice a decreasing trend for IL-10 in the patient groups.

Cytokines operate at hormonal concentrations via high affinity receptors. They are produced on demand and travel only over short distances. The concentrations in vivo are in the range of a few picograms to nanograms per milliliter. As a result of this localized action at extremely low levels, their serum concentrations may not reliably reflect regional activation. Opposed to hormones, cytokines are pleiotropic and redundant. This fact may explain the heterogeneity of the obtained results (37).

Most post-traumatic inflammatory changes noticed in CRPS patients are mediated by two neuropeptides - CGRP and SP. The CGRP (38) and SP (32) serum levels that we found were significantly higher in CRPS patients than in healthy controls.

SP belongs to the family of neurokinins. It binds to different neurokinin receptors and is generally found in both the central and peripheral nervous system. SP is implicated in various diseases such as asthma, atopic dermatitis and inflammatory bowel disease $(39,40)$. It is released in the skin from sensory neurons, endothelial cells, dendritic cells, monocytes/macrophages, polymorphonuclear leukocytes and lymphocytes (41-45). It induces vessel relaxation and extravasation of plasma proteins $(46,47)$. 
The results of our study are also supported by the findings of Weber et al. (48), who demonstrated the increased release of neuropeptides in patients with CRPS. The same modifications resulting in severe extravasation of plasma proteins was observed after strong transcutaneous electrical stimulation or the administration of exogenous SP (48).

We found significantly increased SP levels $(p<0.0001)$ in the CRPS patients. Our data, linked to the literature, provides proof that SP might be involved in CRPS. It might be partly responsible for the symptoms of CRPS through neuromodulating and immunomodulating properties.

The other neuropeptide, the CGRP, a member of the calcitonin family, was also found to be significantly higher $(p<0.0001)$ in patients with CRPS. CGRP is the major transmitter that induces the neurogenic vasodilatation of arterioles. This is mainly due to its actions on the endothelial cells and vascular smooth muscles (49). Features frequently seen in CRPS like increased hair growth and sweat gland activation are also stimulated by CGRP (25). Cell types participating in both innate (dendritic cells, keratinocytes, mast cells) and adaptive ( $\mathrm{T}$ lymphocytes) immunity are directly attracted and activated by SP and CGRP (49). We obtained higher levels of neuropeptides in patients with acute CRPS and slightly lower concentrations in chronic CRPS than in the acute group. It is likely that in patients with chronic CRPS, central mechanisms play a role in maintaining at least the sensory symptoms (50). This was reported by other studies as well (38).

ROC analysis has lately become a common method for evaluating the accuracy of medical diagnostic systems. The area under the curve (AUC) establishes the essential ability of the test to distinguish between the healthy population and patients (51). Using this method as a measure of diagnostic performance, one can compare individual tests or judge whether the various combination of tests can improve diagnostic accuracy. We exhibited the ROC curves only for the neuropeptides (SP and CGRP, acute and chronic) since only those showed statistically significant differences (Table 2, Figure 4) (52).

Table 2. AUC and $P$ value afferent to the ROC curves

\begin{tabular}{|l|c|c|c|c|}
\hline \multirow{2}{*}{} & \multicolumn{2}{|c|}{ SP } & \multicolumn{2}{c|}{ CGRP } \\
\cline { 2 - 5 } & Acute & Chronic & Acute & Chronic \\
\hline AUC & 0.8475 & 0.7169 & 0.8550 & 0.7131 \\
\hline P value & 0.0002 & 0.0065 & 0.0001 & 0.0075 \\
\hline
\end{tabular}

ROC curve of SP (Acute)

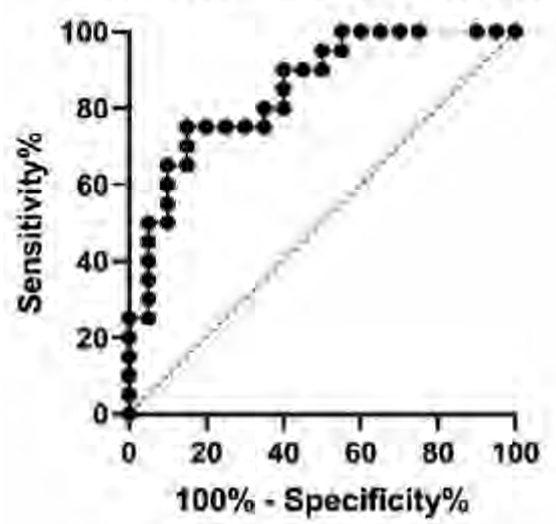

ROC curve of CGRP (Acute)

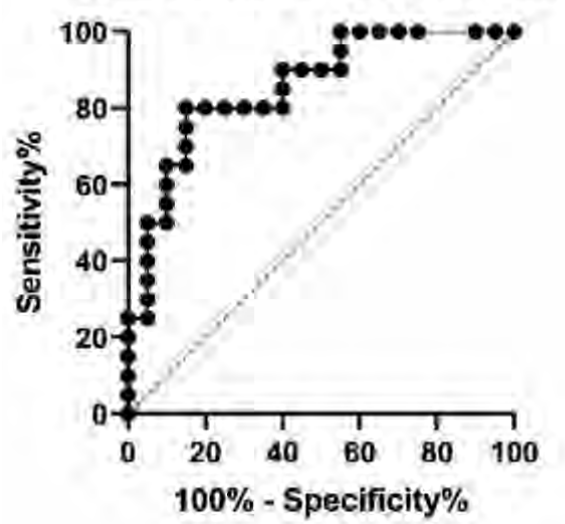

ROC curve of SP (Chronic)

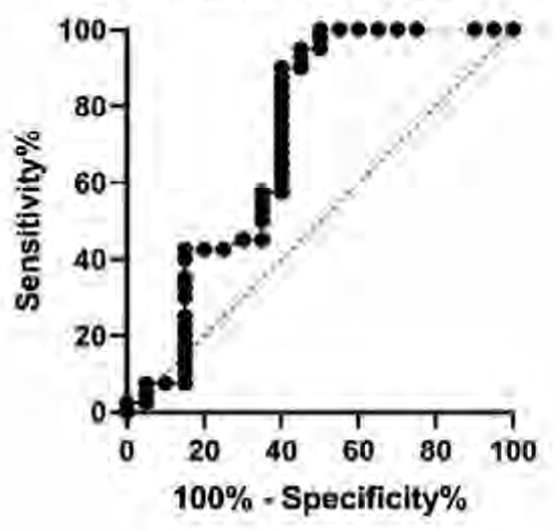

ROC curve of CGRP (Chronic)

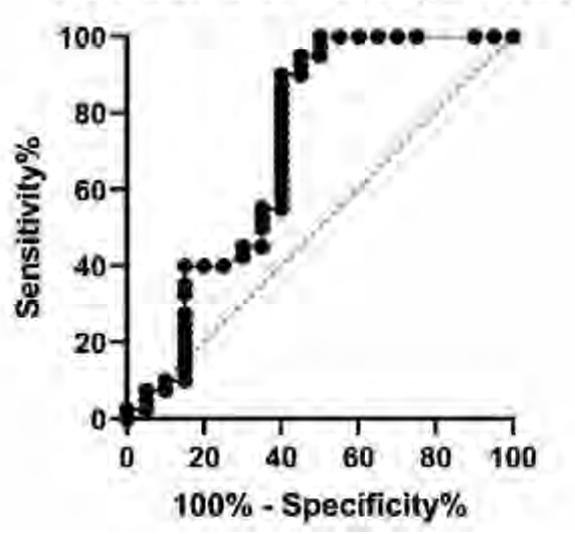

FIGURE 4. ROC curves for $S P$ and $C G R P$ 
Given the results we obtained we can draw the conclusion that the neuropeptides might be useful to support the CRPS diagnostic, as it is a neurogenic condition. Even though the cytokine profile did not return significant differences between control and diseased groups this might be explained by the fact that higher cytokine levels indicate a more systemic inflammation rather than a regional (local) neurogenic inflammation.

\section{CONCLUSIONS}

The results we obtained are consistent with other published findings regarding the inflammatory marker profile in CRPS. This disease can be a debilitating condition, and a careful clinical examination and an accurate diagnostic protocol might have direct implications for understanding mechanisms and for designing optimal treatment protocols. In the present study we tried to establish which inflamma- tory markers (SP, CGRP, TNF $\alpha$, IL-1 $\beta$, IL-6, IL-10) could be successfully used in order to help confirm the CRPS diagnostic. Our results indicated that the best markers were, actually, the neuropeptides (SP and CGRP). In order to support this statement, we used the ROC curve method. Even though our study provides evidence for increased neuropeptide release and neurogenic inflammation in CRPS, this does not mean that the other factors were entirely insignificant. One meta-analysis (53) on CRPS studies reveals a relatively high degree of heterogeneity regarding inflammation markers, especially cytokines as they act at extremely low concentrations. We consider that further longitudinal studies in patients with CRPS will be important to improve our understanding of this complex process. Including more patients in the acute phase might offer some insight on the cytokines' role, as they could be increased more frequently in comparison to chronic patients, due to their short half-life.

Conflict of interest: none declared Financial support: none declared

\section{REFERENCES}

1. Bruehl S, Chung O. Complex regional pain syndrome, diagnosis and pathophysiology of. In Aminoff M, Daroff R (eds): Encycl. Neurol. Sci., San Diego: Academic Press, 2003:749-754.

2. IASP Task Force on Taxonomy. Classification of chronic pain, second edition. Descriptions of chronic pain syndromes and definitions of pain terms, Seattle: IASP Press, 1994.

3. de Mos M, de Bruijn A, Huygen F et al. The incidence of complex regional pain syndrome: A population-based study. Pain 2007; 129(1):12-20.

4. Sandroni P, Benrud-Larson L, McClelland R, Low P. Complex regional pain syndrome type I: Incidence and prevalence in Olmsted county, a population-based study. Pain 2003; 103(1-2):199-207.

5. Harden N, Bruehl S, Galer B et al. Complex regional pain syndrome: Are the IASP diagnostic criteria valid and sufficiently comprehensive? Pain 1999; 83(2):211-219.

6. Bruehl S, Husfeldt B, Lubenow T et al. Psychological differences between reflex sympathetic dystrophy and non-RSD chronic pain patients. Pain 1996; 67(1):107-114.

7. Geertzen J, Dijkstra P, Groothoff J et al. Reflex sympathetic dystrophy of the upper extremity-a 5.5-year follow-up. Part I. Impairments and perceived disability. Acta Orthop. Scand. 1998; 69(sup279):12-18.

8. Geertzen J, Dijkstra P, Groothoff J et al. Reflex sympathetic dystrophy of the upper extremity-a 5.5-year follow-up. Part II. Social life events, general health and changes in occupation. Acta Orthop. Scand. 1998; 69(sup279):19-23.

9. Geertzen J, Dijkstra P, van Sonderen E et al. Relationship between impairments, disability and handicap in reflex sympathetic dystrophy patients: A long-term follow-up study. Clin. Rehabil. 1998; 12(5):402-412.

10. 1 Kemler $\mathrm{M}$, de Vet $\mathrm{H}$. Health-related quality of life in chronic refractory reflex sympathetic dystrophy (complex regional pain syndrome type I). J. Pain Symptom Manage. 2000; 20(1):68-76.

11. Quisel A, Gill J, Witherell P. Complex regional pain syndrome underdiagnosed. J. Fam. Pract. 2005; 54(6):524-532.

12. Burton A, Bruehl S, Harden R. Current diagnosis and therapy of complex regional pain syndrome: Refining diagnostic criteria and therapeutic options. Expert Rev. Neurother. 2005; 5(5):643-651.

13. Chung O, Bruehl S. Complex regional pain syndrome. Curr. Treat. Options Neurol. 2003; 5(6):499-511.

14. Kingery W. A critical review of controlled clinical trials for peripheral neuropathic pain and complex regional pain syndromes. Pain 1997; 73(2):123-139.

15. Perez R, Kwakkel G, Zuurmond W, de Lange J. Treatment of reflex sympathetic dystrophy (CRPS type 1): A research synthesis of 21 randomized clinical trials. J. Pain Symptom Manage. 2001; 21(6):511-526.

16. Baron $\mathrm{R}$, Fields $\mathrm{H}$, Jänig $\mathrm{W}$ et al. National institutes of health workshop: Reflex sympathetic dystrophy/complex regional pain syndromes - State-of-the-science. Anesth. Analg. 2002; 95(6):1812-1816.

17. Harden N, Bruehl S, Perez R et al. Validation of proposed diagnostic criteria (the "Budapest Criteria") for complex regional pain syndrome. Pain 2010; 150(2):268-274.

18. Harden R, Bruehl S. Diagnostic criteria: The statistical derivation of the four criterion factors. In Wilson P, Stanton-Hicks M, Harden R (eds): CRPS Curr. diagnosis Ther., Seattle: IASP Press, 2005:45-58.

19. Braus D, Krauss J, Strobel J. The shoulder-hand syndrome after stroke: A prospective clinical trial. Ann. Neurol. 1994; 36(5):728733.

20. Christensen K, Jensen E, Noer I. The reflex dystrophy syndrome response to treatment with systemic corticosteroids. Acta Chir. Scand. 1982; 148(8):653-655.

21. Cheng J, Ji R. Intracellular signaling in primary sensory neurons and persistent pain. Neurochem. Res. 2008; 33(10):1970-1978.

22. Üçeyler N, Eberle T, Rolke R et al. Differential expression patterns of cytokines in complex regional pain syndrome. Pain 2007; 132(1-2):195-205.

23. Birklein F. Complex regional pain syndrome. J. Neurol. 2005; 252(2):131-138. 
24. Ferreira S, Lorenzetti B, Poole S. Bradykinin initiates cytokinemediated inflammatory hyperalgesia. Br. J. Pharmacol. 1993; 110(3):1227-1231.

25. Birklein F, Schmelz M. Neuropeptides, neurogenic inflammation and complex regional pain syndrome (CRPS). Neurosci. Lett. 2008; 437(3):199-202.

26. Schlereth T, Dittmar J, Seewald B, Birklein F. Peripheral amplification of sweating - a role for calcitonin gene-related peptide. J. Physiol. 2006; 576(3):823-832

27. Backonja M. Defining neuropathic pain. Anesth. Analg. 2003; 97(3):785-790.

28. Krause S, Backonja M. Development of a neuropathic pain questionnaire. Clin. J. Pain 2003; 19(5):306-314.

29. Daut R, Cleeland C, Flanery R. Development of the Wisconsin Brief Pain Questionnaire to assess pain in cancer and other diseases. Pain 1983; 17(2):197-210.

30. Koch A, Zacharowski K, Boehm O et al. Nitric oxide and proinflammatory cytokines correlate with pain intensity in chronic pain patients. Inflamm. Res. 2007; 56(1):32-37.

31. Üçeyler N, Valenza R, Stock M et al. Reduced levels of antiinflammatory cytokines in patients with chronic widespread pain. Arthritis Rheum. 2006; 54(8):2656-2664.

32. Schinkel C, Gaertner A, Zaspel J et al. Inflammatory mediators are altered in the acute phase of posttraumatic complex regional pain syndrome. Clin. J. Pain 2006; 22(3):235-239.

33. Aderka D. The potential biological and clinical significance of the soluble tumor necrosis factor receptors. Cytokine Growth Factor Rev. 1996; 7(3):231-240.

34. Sommer C. Cytokines and neuropathic pain. In Hansson P, Fields $\mathrm{H}$, Hill R, Marchettini P (eds): Neuropathic pain Pathophysiol. Treat., Seattle: IASP Press, 2001:37-62

35. Eastgate J, Wood N, Di Giovine F et al. Correlation of plasma interleukin 1 levels with disease activity in rheumatoid arthritis Lancet 1988; 332(8613):706-709.

36. Watkins L, Maier S. Implications of immune-to-brain communication for sickness and pain. Proc. Natl. Acad. Sci. 1999; 96(14):7710 7713.

37. Sommer C, Kress M. Recent findings on how proinflammatory cytokines cause pain: Peripheral mechanisms in inflammatory and neuropathic hyperalgesia. Neurosci. Lett. 2004; 361(1-3):184-187.

38. Birklein F, Schmelz M, Schifter S, Weber M. The important role of neuropeptides in complex regional pain syndrome. Neurology 2001; 57(12):2179-2184.

39. Joos G, Germonpre P, Pauwels R. Role of tachykinins in asthma. Allergy 2000; 55(4):321-337.
40. Lecci A, Giuliani S, Tramontana M et al. Peripheral actions of tachykinins. Neuropeptides 2000; 34(5):303-313.

41. Linnik M, Moskowitz M. Identification of immunoreactive substance $\mathrm{P}$ in human and other mammalian endothelial cells. Peptides 1989; 10(5):957-962.

42. Ho W, Lai J, Zhu X et al. Human monocytes and macrophages express substance P and neurokinin-1 receptor. J. Immunol. 1997; 159(11):5654-5660.

43. Lai J, Douglas S, Zhao M, Ho W. Quantification of substance P mRNA in human mononuclear phagocytes and lymphocytes using a mimic-based RT-PCR. J. Immunol. Methods 1999; 230(1-2):149-157.

44. De Giorgio R, Tazzari P, Barbara G et al. Detection of substance P immunoreactivity in human peripheral leukocytes. J. Neuroimmunol. 1998; 82(2):175-181.

45. Lambrecht B, Germonpré P, Everaert E et al. Endogenously produced substance $P$ contributes to lymphocyte proliferation induced by dendritic cells and direct TCR ligation. Eur. J. Immunol. 1999; 29(12):3815-3825.

46. Iwamoto I, Nakagawa N, Yamazaki H et al. Mechanism for substance $\mathrm{P}$-induced activation of human neutrophils and eosinophils. Regul. Pept. 1993; 46(1-2):228-230.

47. Schratzberger P, Reinisch N, Prodinger W et al. Differential chemotactic activities of sensory neuropeptides for human peripheral blood mononuclear cells. J. Immunol. 1997; 158(8):3895-3901.

48. Weber M, Birklein F, Neundörfer B, Schmelz M. Facilitated neurogenic inflammation in complex regional pain syndrome. Pain 2001; 91(3):251-257.

49. Chiu I, von Hehn C, Woolf C. Neurogenic inflammation and the peripheral nervous system in host defense and immunopathology. Nat. Neurosci. 2012; 15(8):1063-1067.

50. Rommel O, Malin J, Zenz M, Jänig W. Quantitative sensory testing, neurophysiological and psychological examination in patients with complex regional pain syndrome and hemisensory deficits. Pain 2001; 93(3):279-293.

51. Metz C. Basic principles of ROC analysis. Semin. Nucl. Med. 1978; 8(4):283-298

52. Hajian-Tilaki K. Receiver operating characteristic (ROC) curve analysis for medical diagnostic test evaluation. Casp. J. Intern. Med. 2013; 4(2):627-635.

53. Parkitny L, McAuley J, Di Pietro F et al. Inflammation in complex regional pain syndrome: A systematic review and meta-analysis. Neurology 2013; 80(1):106-117. 